Research Paper

\title{
The Impact of PD-L1 Expression in Patients with Metastatic GEP-NETs
}

\author{
Seung Tae Kim ${ }^{1 *}$, Sang Yun Ha2 ${ }^{*}$, Sujin Lee ${ }^{1}$, Soomin Ahn², Jeeyun Lee ${ }^{1}$, Se Hoon Park ${ }^{1}$, Joon Oh Park ${ }^{1}$, Ho \\ Yeong Lim ${ }^{1}$, Won Ki Kang${ }^{1}$, Kyoung-Mee Kim ${ }^{\circledR}$, Young Suk Park ${ }^{\circledR}$ \\ 1. Division of Hematology-Oncology, Department of Medicine, \\ 2. Department of Pathology \& Translational Genomics, Samsung Medical Center, Sungkyunkwan University School of Medicine, Seoul, Korea. \\ *S.T.K and S.Y.H. contributed equally to this work. \\ $\square$ Corresponding authors: Young Suk Park, MD. PhD. Division of Hematology/Oncology, Department of Medicine, Samsung Medical Center, Sungkyunkwan \\ University School of Medicine, 81 Irwon-ro, Gangnam-gu, Seoul 135-710, Korea. Tel.: +82-2-3410-3451, Fax: +82-2-3410-1754 Email: pys27hmo@skku.edu Or \\ Kyoung-Mee Kim, MD, PhD. Department of Pathology, Center for Companion Diagnostics, Innovative Cancer Medicine Institute, Samsung Medical Center, \\ Sungkyunkwan University School of Medicine, 81 Irwon-ro, Gangnam-gu, Seoul 135-710, Korea. Tel: +82-2-3410-2807; Fax: +82-2-3410-1754 Email: \\ kkmkys@skku.edu.
}

( ) Ivyspring International Publisher. Reproduction is permitted for personal, noncommercial use, provided that the article is in whole, unmodified, and properly cited. See http://ivyspring.com/terms for terms and conditions.

Received: 2015.09.01; Accepted: 2015.12.08; Published: 2016.02.05

\begin{abstract}
Programmed death-ligand 1 (PD-LI), which is expressed on many cancer cells, interacts with PDI expressed on the surface of $T$ cells, inhibiting the $T$ cells and blocking the antitumor immune response. Expression of PD-LI in gastroenteropancreatic neuroendocrine tumors (GEP-NETs) has not been studied. We investigated the impact of PD-L1 expression in 32 patients with metastatic GEP-NET.

The expression of PD-LI was evaluated using an anti-PD-LI immunohistochemistry (IHC) antibody optimized for staining of formalin-fixed paraffin-embedded (FFPE) tissue samples. The correlation between PD-LI and clinicopathological data including survival and response to systemic treatments was analyzed.

Primary sites were 24 foregut-derived GEP-NETs, including stomach $(n=1)$, duodenum $(n=2)$, biliary tract $(n=7)$, and pancreas $(n=14)$, and 8 hindgut-derived GEP-NETs of the distal colon and rectum. Among the 32 patients with metastatic GEP-NET analyzed in this study, 7 (21.9\%) had expression of PD-L1 in tumor tissues. Expression of PD-L1 was significantly associated with high-grade WHO classification (grade 3$)(p=0.008)$ but not with gender, primary site, and number of metastatic sites $(p>0.05)$. The status of PD-LI expression was statistically associated with progression-free survival (PFS) for first-line systemic treatment $(\mathrm{p}=0.047)$. Moreover, the status of PD-LI expression could significantly predict overall survival $(p=0.037)$.

The expression of PD-LI was associated with higher WHO tumor grade (grade 3 ) in metastatic GEP-NETs. PD-L1 expression had both predictive and prognostic value for survival of patients with metastatic GEP-NETs.
\end{abstract}

Key words: Programmed death-ligand 1 (PD-L1), Gastroenteropancreatic neuroendocrine tumor (GEP-NET), WHO classification.

\section{Introduction}

The immune system influences the fate of developing cancers by functioning not only as a tumor promoter that facilitates cellular transformation, promotes tumor growth, and sculpts tumor cell immunogenicity [1-6], but also as an extrinsic tumor suppressor that either destroys developing tumors or restrains their expansion [2, 5, 7]. Programmed death-ligand 1 (PD-L1), which is expressed on many cancer and immune cells, plays an important role in blocking the cancer immunity cycle by binding pro- 
grammed death-1 (PD-1) and B7.1, both of which are negative regulators of $\mathrm{T}$ lymphocyte activation [8]. Indeed, tumor cells from different locations express PD-L1 and thus can inhibit the immune response. Recently, clinical trials testing anti-PD-L1 or anti-PD1 agents for restoration of antitumor immunity have reported very promising outcomes, notably in melanoma and renal, lung, prostate, and bladder cancer. PD-L1 expression has been studied in different cancers including kidney, lung, pancreas, esophagus, ovary, colorectal, head and neck, breast, and skin (melanoma), with evidence of correlations with clinicopathological tumor features in several studies [9-15]. Neuroendocrine tumors (NET) are a heterogeneous group of malignancies derived from neuroendocrine cell compartments with roles in both the endocrine and the nervous system. The majority of NETs are gastroenteropancreatic (GEP) in origin, arising in the foregut, midgut, or hindgut [16]. Although NETs are known to be very rare [17], recent studies based on the Surveillance, Epidemiology and End Results (SEER) cancer registry and European studies demonstrated an increasing incidence of NETs [17]. Moreover, a Korean study showed a remarkable increase in the incidence of GEP-NETs during the last decade [18]. Although GEP-NETs are increasingly being diagnosed, a concomitant improvement in outcomes has not been noted. Recently, two molecularly targeted agents, sunitinib and everolimus, have been approved for advanced pancreatic NETs [19, 20] and might prolong the survival of patients with GEP-NET. However, most GEP-NET patients experience resistance to these treatments and additional effective treatment options are very limited [21]. Cancer immunotherapy is presently one of the areas in which major medical breakthroughs are being witnessed with impressive results reported by several groups $[8,22]$. One key target of cancer immunotherapies is the PD1-PD-L1 pathway; however, this pathway has not been studied in metastatic

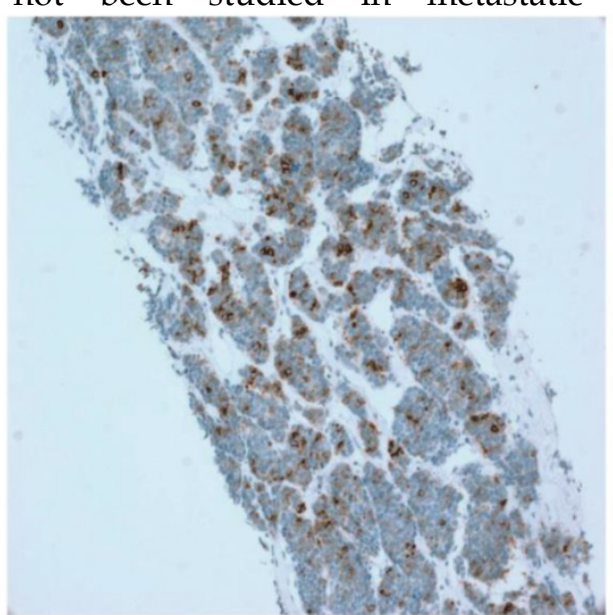

GEP-NETs. Here, we investigated the impact of PD-L1 expression on survival in 32 patients with metastatic GEP-NET and searched for correlations between PD-L1 expression and clinicopathlogic data.

\section{Material and methods}

\section{Patients}

Among patients who were pathologically diagnosed with metastatic GEP-NETs in Samsung Medical Center between June 2011 and September 2014, 32 were analyzed for PD-L1 expression in this study. The following clinicopathological characteristics were collected for all 32 patients: age, gender, primary site, tumor grade according to the 2010 WHO classification, liver metastasis, number of metastatic sites, site of metastasis, and information on chemotherapy.

\section{Immunohistochemistry (IHC) of PD-LI}

Tumor sections were freshly cut to $4 \mathrm{~mm}$ and dried at $60^{\circ} \mathrm{C}$ for 30 minute. PDL- 1 immunohistochemistry (rabbit anti-human PDL-1 monoclonal, 1:25, clone SP142; Ventana, Tuscon, AZ) was performed on an automated immunostainer (Benchmark; Ventana). Antigen retrieval was performed for $92 \mathrm{~min}$ with $\mathrm{CC} 1$ and the antibody was incubated for $120 \mathrm{~min}$ un $37^{\circ} \mathrm{C}$ in Ventana BencaMark XT. Signal visualization was achieved with the Optiview DAB IHC detection kit (Catalogue number 760-700) and Optiview Amplification kit (Catalogue number 860-099).

PD-L1 expression was evaluated on tumor cells. Tumor infiltrating immune cells are not identified in all cases. The proportion of PD-L1-positive cells was estimated as the percentage of total tumor cells; tumor cells typically showed membranous staining with a variably component of cytoplasmic staining. Specimens were categorized as IHC negative or positive if $<1 \%$ or $\geq 1 \%$ of cells were stained by PD-L1 mAb, respectively (Figure 1 ).

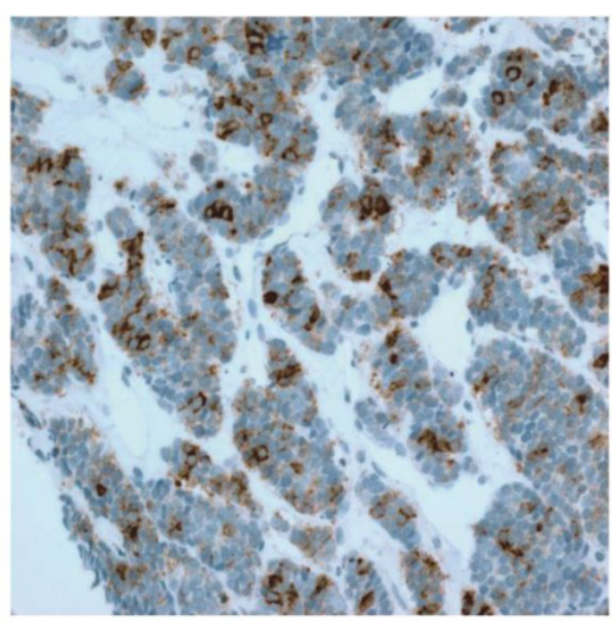

Figure 1. Representative image of PD-L1 IHC staining of tumors from patients with GEP-NETs 


\section{Statistical analyses}

Correlation between the status of PD-L1 expression and clinicopathological variables was analyzed using the t-test or the Fisher's exact test as appropriate or one-way analysis of variance. Treatment outcomes were estimated as response rate (RR), disease control rate (DCR), progression-free survival (PFS), and overall survival (OS). PFS and OS were defined as the time from the first study treatment to the date of disease progression or death, respectively. Descriptive statistics were reported as proportions and medians. Kaplan-Meier estimates were used in the analysis of all time to event variables, and the 95\% confidence interval (CI) for the median time to event was computed.

\section{Results}

\section{Patient characteristics}

Among patients who were pathologically diagnosed with metastatic GEP-NET in Samsung Medical Center between June 2011 and September 2014, 32 were analyzed for PD-L1 expression in this study. Baseline characteristics of these 32 patients are listed in Table 1. The median age of the patients was 60.0 years (range, 35-88) and the male to female ratio was 1.29. Primary sites included 24 foregut-derived GEP-NETs [stomach $(n=1)$, duodenum $(n=2)$, biliary tract $(n=7)$, and pancreas $(n=14)]$ and 8 hindgut-derived GEP-NETs of distal colon and rectum. According to WHO classification, 17 patients had grade 3 neuroendocrine carcinoma (NEC) and 15 had grade 2 NET. All 32 patients had liver metastases. Twenty-one patients $(65.6 \%)$ had 2 or more metastatic sites. The majority $(78.1 \%)$ of patients received the recommended systemic treatment after the diagnosis of metastatic GEP-NETs. However, 7 patients (21.9\%) were managed by only best supportive care because of patient preference or poor performance status (PS).

\section{The correlation between PD-LI expression and clinicopathological variables}

Among the 32 patients with metastatic GEP-NET analyzed for PD-L1 expression, 7 (21.9\%) exhibited expression of PD-L1 in tumor tissues. The expression of PD-L1 was significantly associated with high-grade WHO classification (grade 3$)(p=0.008)$ but not with gender, primary site, or number of metastatic sites ( $>0.05)$ (Table 2). Among 21 patients receiving the recommended systemic therapies as first line, 4 patients had PD-L1-positive tumors and 17 patients had PD-L1-negative tumors. There was significant difference in the tumor response according to the status of PD-L1 (Table 3).
Table 1. Baseline characteristics in 32 gastroenteropancreatic neuroendocrine tumors (GEP-NETs) patients.

\begin{tabular}{lll}
\hline Variables & $\mathrm{n}$ & $\%$ \\
\hline Gender & 18 & 56.3 \\
Male & 14 & 43.7 \\
Female & & \\
Age, years & $60,(35-88)$ & \\
Median, range & & \\
Primary tumor site & 1 & 3.1 \\
Stomach & 2 & 6.2 \\
Duodenum & 6 & 18.8 \\
Gall Bladder & 1 & 3.1 \\
Biliary & 14 & 43.8 \\
Pancreas & 8 & 25.0 \\
Rectum & & \\
WHO classification & 15 & 46.9 \\
Grade II neuroendocrine tumor & 17 & 53.1 \\
Grade III neuroendocrine carcinoma & & \\
Liver metastasis & 32 & 100.0 \\
Yes & & \\
Number of metastatic sites & 11 & 34.4 \\
1 & 21 & 65.6 \\
$2 \leq$ & & \\
First line treatment & 7 & 21.9 \\
Only supportive care & & \\
Systemic treatment & & \\
\hline
\end{tabular}

Table 2. The difference of PDL1 expression by immunohistochemistry (IHC) according to clinical characteristics.

\begin{tabular}{|c|c|c|c|}
\hline \multicolumn{4}{|c|}{ (A) Gender (Male vs Female), p=1.000 } \\
\hline \multicolumn{4}{|c|}{ PDL1 IHC } \\
\hline Gender & Positive & Negative & Total \\
\hline Male & 4 & 14 & 18 \\
\hline Female & 3 & 11 & 14 \\
\hline Total & 7 & 25 & 32 \\
\hline \multirow{2}{*}{\multicolumn{4}{|c|}{$\begin{array}{l}\text { (B) Primary site (Foregut vs Midgut vs } \\
\text { Hindgut), } \mathrm{p}=\mathbf{1 . 0 0 0} \\
\text { PDL1 IHC }\end{array}$}} \\
\hline & & & \\
\hline Foregut & 5 & 19 & 24 \\
\hline Hindgut & 2 & 6 & 8 \\
\hline Total & 7 & 25 & 32 \\
\hline \multirow{2}{*}{\multicolumn{4}{|c|}{$\begin{array}{l}\text { (C) WHO classification (Grade II vs } \\
\text { Grade III), } \mathbf{p}=\mathbf{0 . 0 0 8} \\
\text { PDL1 IHC }\end{array}$}} \\
\hline PDL1 IHC & & & \\
\hline WHO & Positive & Negative & Total \\
\hline Grade II & 0 & 15 & 15 \\
\hline Grade III & 7 & 10 & 17 \\
\hline Total & 7 & 25 & 32 \\
\hline \multicolumn{4}{|c|}{$\begin{array}{l}\text { (D) Number of metastatic sites (1 vs } 2 \leq) \text {, } \\
p=0.719\end{array}$} \\
\hline \multicolumn{4}{|l|}{ PDL1 IHC } \\
\hline Metastatic sites & Positive & Negative & Total \\
\hline 1 & 2 & 9 & 11 \\
\hline $2 \leq$ & 5 & 16 & 21 \\
\hline Total & 7 & 25 & 32 \\
\hline
\end{tabular}

\section{The impact of PD-LI on patient survival}

In 21 patients receiving systemic therapies as first line, the median progression-free survival (PFS) was 4.9 months (95\% CI 4.2-5.5). There was a significant difference in PFS between patients who were positive and negative for PD-L1 (4.2 vs. 5.1 months, $\mathrm{p}=0.047$ ) (Figure 2). The median overall survival (OS) 
was 17.3 months (95\% CI 11.7-22.8) for all 32 patients. According to the status of PD-L1, median OS was 16.0 months (95\% CI 7.7-24.1) in patients with PD-L1-positive tumors and 24.8 months $(95 \%$ CI 2.6-46.9) in those with PD-L1-negative tumors $(\mathrm{p}=0.037)$ (Figure 3$)$.

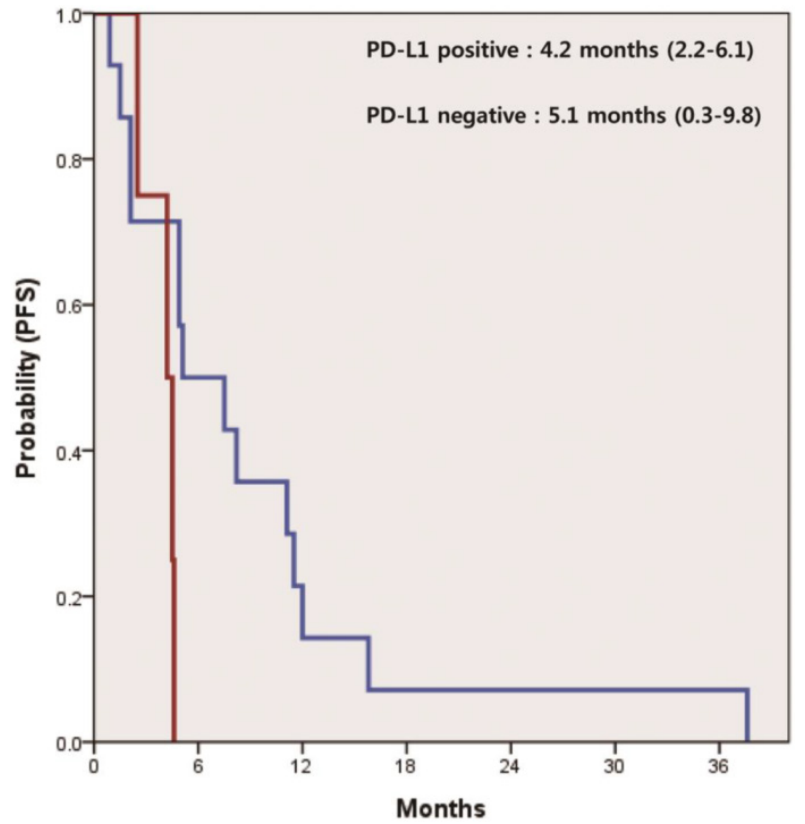

Figure 2. Kaplan-Meier curve of progression free survival (PFS) for systemic treatment of $1^{\text {st }}$ line by PD-L1 IHC status $(p=0.047)$.

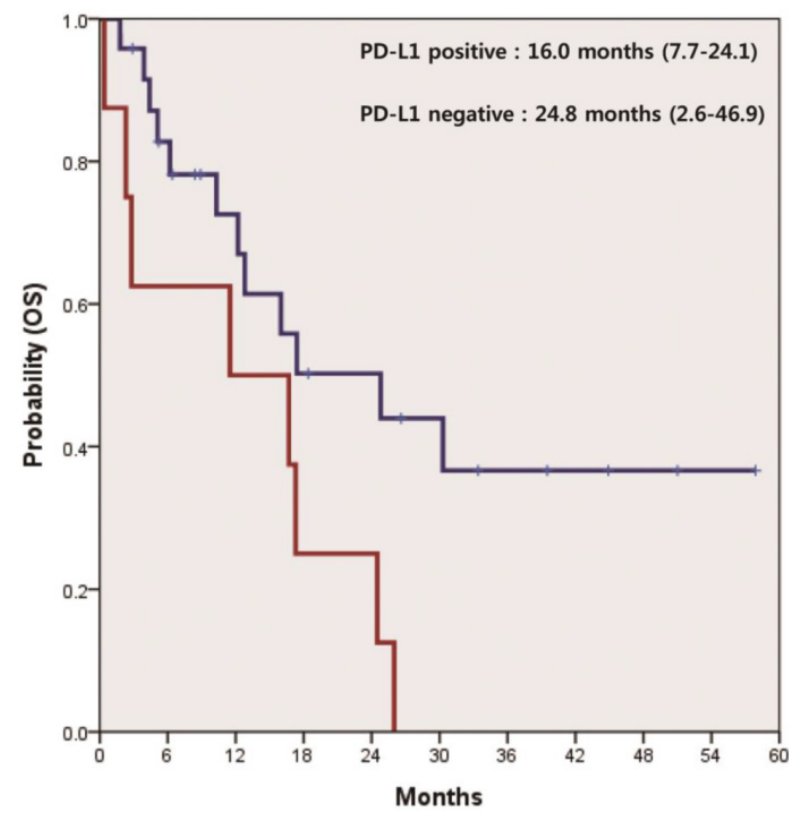

Figure 3. Kaplan-Meier curve of overall survival (OS) by PD-L1 IHC status $(p=0.037)$.
Table 3. The difference of response for $1^{\text {st }}$ line treatment according to PD-LI expression by immunohistochemistry (IHC) $p=0.028, p=1.000$.

\begin{tabular}{lll}
\hline & PD-L positive & PD-L1 negative \\
\hline 1st line chemotherapy & $\mathrm{N}=4$ & $\mathrm{~N}=17$ \\
Partial response & 3 & 2 \\
Stable disease & 0 & 9 \\
Progressive disease & 1 & 5 \\
Response rate & $75.0 \%$ & $11.8 \%$ \\
Disease control rate & $75.0 \%$ & $64.7 \%$ \\
\hline
\end{tabular}

\section{Discussion}

Inhibition of PD-L1 is a promising novel target in cancer immunotherapy [23]. This study analyzed a heterogeneous collection of metastatic GEP-NETs for expression of PD-L1. We aimed to investigate the role of PD-L1 expression as a predictive and prognostic marker for survival in 32 patients with metastatic GEP-NET. We also searched for correlations between PD-L1 expression and clinicopathlogic data. This analysis revealed that the expression of PD-L1 was significantly associated with high-grade WHO classification (grade 3) $(p=0.008)$ but not with gender, primary site, and the number of metastatic sites ( $p>0.050$ ). Moreover, PD-L1 might be a useful predictive and prognostic biomarker for survival in metastatic GEP-NETs. To our knowledge, this is the first report evaluating the characteristics and value of PD-L1 expression in patients with metastatic GEP-NET.

PD-L1 expression has been studied in many cancers [9-15]. Studies of breast cancer showed that the expression of PD-L1 was associated with poor prognosis features: large tumor size, high grade, negative hormone receptor status, positive HER2 status, and high proliferative rate [24]. The characteristics of indolent or aggressive GEP-NET were determined by tumor grade or differentiation [17, 25]. In general, tumor grade or differentiation has been based on the World Health Organization (WHO) classification. The 2010 WHO classification divides NETs into two main subgroups, tumor (NET) and carcinoma (NEC), according to Ki67 value and mitotic count. NET is defined as $<20 \% \mathrm{Ki} 67$ and/or $<20 /$ high power field (HPF) mitotic count, and is further sub-classified into Grade 1 (G1) (Ki67 $\leq 2$ and/or mitosis $\leq 5$ ) and Grade 2 (Ki67 3-20\% and/or mitosis 5-20/HPF) [26]. All NETs with Ki67 $>20 \%$ and/or mitosis > 20/HPF are Grade 3 tumors (NECs), which includes small-cell or large-cell carcinoma. Thus, grade 3 GEP-NETs have more proliferative and aggressive clinicopathologic features. Our finding that expression of PD-L1 was related to a more proliferative and aggressive grade 3 type of GEP-NET was consistent with previous studies [24, 27, 28]. This correlation between the expression of 
PD-L1 and more proliferative tumor type might be caused by the higher aberration rate of hyperproliferative tumor cells, which is responsible for higher immunogenicity due to the rapid appearance of neoantigens.

Anti-PD-L1 therapies are under the spotlight in oncology as next-generation anticancer agents. Herbst et al. showed that anti-PD-L1 therapy (MPDL3280A) was safe and active in several cancer types, including non-small cell lung cancer, melanoma, renal cell carcinoma, and other solid tumors [8]. Powles et al. also reported that MPDL3280A was a new therapeutic option for patients with bladder cancer. In these two studies, tumor expression of PD-L1 was associated with a higher response to anti-PD-L1 therapy [22]. In our study, 7 of $32(21.9 \%)$ patients had expression of PD-L1 in tumor tissues. In particular, in the subgroup of grade 3 GEP-NETs 7 of 17 (41.2\%) patients had tumor expression of PD-L1. There are limited therapeutic options for grade 3 GEP-NET (NEC). The high proportion of PD-L1 expression in grade 3 GEP-NETs suggests that anti-PD-L1 therapy may be a useful new therapeutic modality for this subgroup of GEP-NETs. However, although overexpression of PD-L1 might serve as a predictor of tumors that have successfully evaded an immune response, nivolumab, anti-PD1 antibody, could alleviate inhibitory T-cell signaling independently of tumoral PD-L1 expression [29]. In view of similar observations of clinical activity of anti-PD1/PD-L1 therapeutic antibodies in PD-L1 negative patients [30-32], PD-L1 expression might not be necessary for achieving objective response with these agents.

Our study was a retrospective analysis with a small sample size. In addition, there was a lack of standardization of PD-L1 IHC, notably in terms of specificity and reproducibility of available antibodies and the definition of optimal positivity cut-off and interpretative subjectivity. Nevertheless, this analysis identified clinicopathologic characteristics related to expression of PD-L1 in metastatic GEP-NETs and evaluated the predictive and prognostic values of PD-L1 in GEP-NETs for survival. These data may provide useful information and background for future research for immunotherapy for GEP-NET.

\section{Acknowledgement}

This work was supported by a grant from the 20 by 20 project of Samsung Medical Center (GF01140111).

\section{Author contributions}

Seung Tae Kim, and Sang Yun Ha served as co-first authors, each with equal contribution to the manuscript.
Study concept and design: Seung Tae Kim, and Young Suk Park.

Acquisition, analysis or interpretation of data: Seung Tae Kim, Sujin Lee, Jeeyun Lee, Joon Oh Park, Se Hoon Park, Ho Yeong Lim, Won Ki Kang and Young Suk Park.

Draft of the manuscript: Seung Tae Kim and Sang Yun Ha.

Administrative, technical, or material support: Seung Tae Kim, Sang Yun Ha, Soomin Ahn, and Kyoung-Mee Kim.

Study supervision: Kyoung-Mee Kim, and Young Suk Park.

Kyoung-Mee Kim, and Young Suk Park had full access to all the data in the study and take responsibility for the integrity of the data and the accuracy of the data analysis.

\section{Competing Interests}

The authors have declared that no competing interest exists.

\section{References}

1. Coussens LM, Zitvogel L, Palucka AK. Neutralizing tumor-promoting chronic inflammation: a magic bullet? Science. 2013; 339: 286-291.

2. Dunn GP, Bruce AT, Ikeda $\mathrm{H}$, et al. Cancer immunoediting: from immunosurveillance to tumor escape. Nat Immunol. 2002; 3: 991-998.

3. Grivennikov SI, Greten FR, Karin M. Immunity, inflammation, and cancer. Cell. 2010; 140: 883-899.

4. Mantovani A, Allavena P, Sica A, et al. Cancer-related inflammation. Nature. 2008; 454: 436-444

5. Shankaran V, Ikeda H, Bruce AT, et al. IFNgamma and lymphocytes prevent primary tumour development and shape tumour immunogenicity. Nature. 2001; 410: 1107-1111.

6. Trinchieri G. Cancer and inflammation: an old intuition with rapidly evolving new concepts. Annu Rev Immunol. 2012; 30: 677-706.

7. Koebel CM, Vermi W, Swann JB, et al. Adaptive immunity maintains occult cancer in an equilibrium state. Nature. 2007; 450: 903-907.

8. Herbst RS, Soria JC, Kowanetz M, et al. Predictive correlates of response to the anti-PD-L1 antibody MPDL3280A in cancer patients. Nature. 2014; 515: 563-567.

9. Badoual C, Hans S, Merillon N, et al. PD-1-expressing tumor-infiltrating T cells are a favorable prognostic biomarker in HPV-associated head and neck cancer. Cancer Res. 2013; 73: 128-138.

10. Droeser RA, Hirt C, Viehl CT, et al. Clinical impact of programmed cell death ligand 1 expression in colorectal cancer. Eur J Cancer. 2013; 49: 2233-2242.

11. Konishi J, Yamazaki K, Azuma M, et al. B7-H1 expression on non-small cell lung cancer cells and its relationship with tumor-infiltrating lymphocytes and their PD-1 expression. Clin Cancer Res. 2004; 10: 5094-5100.

12. Nomi $T$, Sho $M$, Akahori $T$, et al. Clinical significance and therapeutic potential of the programmed death-1 ligand/programmed death-1 pathway in human pancreatic cancer. Clin Cancer Res. 2007; 13: 2151-2157.

13. Ohigashi $\mathrm{Y}$, Sho $\mathrm{M}$, Yamada $\mathrm{Y}$, et al. Clinical significance of programmed death-1 ligand-1 and programmed death-1 ligand-2 expression in human esophageal cancer. Clin Cancer Res. 2005; 11: 2947-2953.

14. Spranger S, Spaapen RM, Zha Y, et al. Up-regulation of PD-L1, IDO, and $\mathrm{T}(\mathrm{regs})$ in the melanoma tumor microenvironment is driven by CD8(+) $\mathrm{T}$ cells. Sci Transl Med. 2013; 5: 200ra116.

15. Thompson RH, Dong H, Lohse CM, et al. PD-1 is expressed by tumor-infiltrating immune cells and is associated with poor outcome for patients with renal cell carcinoma. Clin Cancer Res. 2007; 13: 1757-1761.

16. Williams ED, Sandler M. The classification of carcinoid tumours. Lancet. 1963; 1: 238-239.

17. Yao JC, Hassan M, Phan A, et al. One hundred years after "carcinoid": epidemiology of and prognostic factors for neuroendocrine tumors in 35,825 cases in the United States. J Clin Oncol. 2008; 26: 3063-3072.

18. Gastrointestinal Pathology Study Group of Korean Society of Pathologists, Cho MY, Kim JM, et al. Current Trends of the Incidence and Pathological Diagnosis of Gastroenteropancreatic Neuroendocrine Tumors (GEP-NETs) in Korea 2000-2009: Multicenter Study. Cancer Res Treat. 2012; 44: 157-165.

19. Raymond E, Dahan L, Raoul JL, et al. Sunitinib malate for the treatment of pancreatic neuroendocrine tumors. N Engl J Med. 2011; 364: 501-513. 
20. Yao JC, Shah MH, Ito T, et al. Everolimus for advanced pancreatic neuroendocrine tumors. N Engl J Med. 2011; 364: 514-523.

21. Naraev BG, Strosberg JR, Halfdanarson TR. Current status and perspectives of targeted therapy in well-differentiated neuroendocrine tumors. Oncology. 2012; 83: 117-127.

22. Powles T, Eder JP, Fine GD, et al. MPDL3280A (anti-PD-L1) treatment leads to clinical activity in metastatic bladder cancer. Nature. 2014; 515: 558-562.

23. Tumeh PC, Harview CL, Yearley JH, et al. PD-1 blockade induces responses by inhibiting adaptive immune resistance. Nature. 2014; 515: 568-571.

24. Sabatier R, Finetti P, Mamessier E, et al. Prognostic and predictive value of PDL1 expression in breast cancer. Oncotarget. 2015; 6: 5449-5464.

25. Pape UF, Berndt U, Muller-Nordhorn J, et al. Prognostic factors of long-term outcome in gastroenteropancreatic neuroendocrine tumours. Endocr Relat Cancer. 2008; 15: 1083-1097.

26. Bernick PE, Klimstra DS, Shia J, et al. Neuroendocrine carcinomas of the colon and rectum. Dis Colon Rectum. 2004; 47: 163-169.

27. Ghebeh H, Mohammed S, Al-Omair A, et al. The B7-H1 (PD-L1) T lymphocyte-inhibitory molecule is expressed in breast cancer patients with infiltrating ductal carcinoma: correlation with important high-risk prognostic factors. Neoplasia. 2006; 8: 190-198.

28. Ghebeh H, Tulbah A, Mohammed S, et al. Expression of B7-H1 in breast cancer patients is strongly associated with high proliferative Ki-67-expressing tumor cells. Int J Cancer. 2007; 121: 751-758.

29. Brahmer J, Reckamp KL, Baas P, et al. Nivolumab versus Docetaxel in Advanced Squamous-Cell Non-Small-Cell Lung Cancer. N Engl J Med. 2015; 373: 123-135.

30. Grosso, J, Horak, CE, Inzunza, D et al. Association of tumor PD-L1 expression and immune biomarkers with clinical activity in patients (pts) with advanced solid tumors treated with nivolumab (anti-PD-1; BMS-936558; ONO-4538). Proc Am Soc Clin Oncol. 2013; 31: 3016 (abstr).

31. Taube JM, Klein A, Brahmer JR, et al. Association of PD-1, PD-1 ligands, and other features of the tumor immune microenvironment with response to anti-PD-1 therapy. Clin Cancer Res. 2014; 20: 5064-5074.

32. Garon, EB, Gandhi, L, Rizvi, N et al. Antitumor activity of pembrolizumab (Pembro; MK-3475) and correlation with programmed death ligand 1 (PD-L1) expression in a pooled analysis of patients (pts) with advanced non-small cell lung carcinoma (NSCLC). Ann Oncol. 2014; 25: LBA43 (abstr). 